\title{
Cardiac involvement at presentation in patients hospitalized with COVID-19 and their outcome in a tertiary referral hospital in Northern Italy
}

\author{
Stefano Ghio ${ }^{1}$ (1) Enrico Baldi ${ }^{2,3} \cdot$ Alessandro Vicentini $^{2} \cdot$ Marco Vincenzo Lenti $^{4} \cdot$ Antonio Di Sabatino $^{4}$. \\ Angela Di Matteo ${ }^{5}$. Valentina Zuccaro ${ }^{5}$. Davide Piloni ${ }^{6}$. Angelo Corsico ${ }^{6}$ - Massimiliano Gnecchi ${ }^{2,3}$. \\ Francesco Speciale $^{7,8} \cdot$ Anna Sabena ${ }^{7,8} \cdot$ Luigi Oltrona Visconti $^{1}$. Stefano Perlini ${ }^{7,8}$ on behalf of San Matteo COVID \\ Cardiac Injury Task Force
}

Received: 13 June 2020 / Accepted: 4 September 2020

(c) Società Italiana di Medicina Interna (SIMI) 2020, corrected publication 2020

\begin{abstract}
The correlation between myocardial injury and clinical outcome in COVID-19 patients is gaining attention in the literature. The aim of the present study was to evaluate the role of cardiac involvement and of respiratory failure in a cohort of COVID-19 patients hospitalized in an academic hospital in Lombardy, one of the most affected Italian (and worldwide) regions by the epidemic. The study included 405 consecutive patients with confirmed COVID-19 admitted to a medical ward from February 25th to March 31st, 2020. Follow-up of surviving patients ended either at hospital discharge or by July 30th, 2020. Myocardial injury was defined on the basis of the presence of blood levels of hs-TnI above the 99th percentile upper reference limit. Respiratory function was assessed as $\mathrm{PaO}_{2} / \mathrm{FiO}_{2}(\mathrm{P} / \mathrm{F})$ ratio. The primary end-point was death for any cause. During hospitalization, 124 patients died. Death rate increased from $7.9 \%$ in patients with normal hs-TnI plasma levels and no cardiac comorbidity to $61.5 \%$ in patients with elevated hs-TnI and cardiac involvement $(p<0.001)$. At multivariable analysis, older age, $\mathrm{P} / \mathrm{F}$ ratio $<200$ (both $p<0.001$ ) and hs-TnI plasma levels were independent predictors of death. However, it must be emphasized that the median values of hs-TnI were within normal range in non-survivors. Cardiac involvement at presentation was associated with poor prognosis in COVID-19 patients, but, even in a population of COVID-19 patients who did not require invasive ventilation at hospital admission, mortality was mainly driven by older age and respiratory failure.
\end{abstract}

Keywords COVID-19 $\cdot$ Cardiac injury $\cdot$ Italy $\cdot$ Prognosis

Luigi Oltrona Visconti and Stefano Perlini equally contributed as co-last authors.

The members of San Matteo COVID Cardiac Injury Task Force are given in Acknowledgements.

Electronic supplementary material The online version of this article (https://doi.org/10.1007/s11739-020-02493-y) contains supplementary material, which is available to authorized users.

Stefano Ghio

s.ghio@smatteo.pv.it

Extended author information available on the last page of the article

\section{Introduction}

Since the onset of coronavirus disease 2019 (COVID-19) pandemic caused by severe acute respiratory syndrome coronavirus 2 (SARS-CoV-2), a growing body of evidence has shown that patients with confirmed COVID-19 may present elevated blood levels of high-sensitivity TnI (hs-TnI) during hospital stay, which might reflect ischemic cardiovascular complications or acute myocarditis [1-3]. An early experience in Wuhan, China, reported that among 416 hospitalized patients with COVID-19, nearly 20\% had elevated levels of TnI at admission and that the overall mortality in this sub-category of patients was higher compared with that of patients with normal TnI levels (52.1 vs $4.5 \%$, respectively, $p<0.001$ ) [4]. Other smaller cohort studies confirmed the poor prognosis of hospitalized COVID-19 patients with concomitant cardiac disease, with or without elevated plasma 
levels of high-sensitivity troponin [5-7]. On the contrary, in a retrospective, multicenter cohort study including all adult patients with confirmed COVID-19 diagnosis admitted at two Hospitals in Wuhan, hs-TnI values did not correlate with mortality when included in a multivariate analysis [8]. In the same study, the levels of TnI tended to increase during the hospitalization, more specifically around day 15 , particularly in the most severe patients [8]. A few studies, including a metanalysis, support the notion that $\mathrm{TnI}$ levels are significantly increased when SARS-CoV-2 infection is more severe [9-13]. These data raise the question if cardiac injury is a contributory cause of COVID-19 severity or vice versa if the severity of the infection may lead to cardiac damage [14]. In particular, in most of these previous studies, the degree of respiratory failure at admission was not reported.

Accordingly, we aimed to verify the role of cardiovascular comorbidities and of hs-TnI plasma levels and respiratory failure upon admission during the initial phase of the COVID-19 outbreak in Pavia, Italy. Secondary objective was to verify whether the predictors of outcome differ when evaluating death or the combination of death and/or ICU admission.

\section{Materials and methods}

\section{Patients}

Four hundred and five consecutive adult ( $\geq 18$ years old) patients admitted with the suspected diagnosis of COVID19 to the Emergency Department of Fondazione IRCCS Policlinico San Matteo (Pavia, Italy) from February 21st to March 31st 2020 were included in this cohort study. We have also included patients with an initially negative nasopharyngeal swab, but with a clear clinical diagnosis of SARS-CoV-2 infection based on symptoms, radiological and blood test findings, as the diagnosis of COVID-19 remains a hard task to accomplish $[15,16]$. In particular, we included only patients coming from high-risk areas, in whom COVID-19 diagnosis was based on the concomitant presence of flu-like symptoms (fever, cough, sore throat), respiratory failure (tachypnoea, hypoxemia), clear radiological signs of interstitial pneumonia, and lymphopenia with increased lactate dehydrogenase. Other potential causes of interstitial pneumonia were ruled out, including atypical bacteria (Mycoplasma or Chlamydia) or other viruses (i.e., influenza A and B, parainfluenza viruses, syncytial respiratory virus, rhinoviruses, adenovirus, other coronaviruses). Patients who were directly hospitalized in the intensive care unit (ICU) were not included in the present study to avoid the confounding factor of extreme hypoxia at admission on the assessment of determinants of in-hospital prognosis. The review board of the Fondazione Policlinico San Matteo approved the publication of anonymized case series of COVID-19 patients using data collected for routine clinical practice and waived the requirement for a specific informed consent.

Patients or the public were not involved in the design, or conduct, or reporting, or dissemination plans of our research.

\section{Data collection}

Demographic characteristics, medical history and previous therapy [ACE inhibitors (ACEi), Angiotensin II receptor blockers (ARB), betablockers, antiarrhythmics, antiplatelets and anticoagulants, clinical data [including $\mathrm{PaO}_{2} / \mathrm{FiO}_{2}(\mathrm{P} / \mathrm{F})$ ] and laboratory findings (including hs-TnI values) of patients upon admission and their subsequent outcome were retrieved from electronic medical records and collected onto a predefined spreadsheet. Determination of hs-TnI blood levels was performed using one validated assay [17].

A 12-lead electrocardiogram (ECG) was collected within the first $24 \mathrm{~h}$ of admission. Subsequently, an ECG was performed only in the presence of chest pain or other suspicious symptoms. ECG analysis focused on rhythm, heart rate, conduction disturbances and ST-T pathological abnormalities. QT interval was measured in at least two leads (DII and V2, or V5) and corrected using the Bazett formula.

\section{Definitions of cardiac involvement}

Myocardial injury was defined as hs-TnI value above the 99th percentile upper reference limit (URL). History of cardiovascular disease included history of ischemic heart disease, heart failure, valvular heart disease and hypertension. Presence of electrocardiographic abnormality included ST-elevation, ST-depression, negative $\mathrm{T}$ waves and atrial fibrillation (AF).

\section{Clinical outcomes}

We considered two hard outcome endpoints: death due to any cause during hospitalization and the combination of death and/or transfer to intensive care unit (ICU) due to the necessity of invasive mechanical ventilation. The follow-up of surviving patients ended either at hospital discharge or by July 30th, 2020. By this date, only one patient was still hospitalized in ICU, whilst all the other patients either died during hospitalization or were discharged.

\section{Statistical analysis}

All data were entered in anonymous form into a database (Microsoft Excel 2019) and then analyzed with MedCalc version 19.2 (MedCalc Software bvba). Categorical variables were presented as number and percentage and 
compared with Chi-square test. Continuous variables were tested for normality with the D'Agostino-Pearson's test. Normally distributed continuous variables were presented as mean \pm standard deviation and compared with $T$ Student's test. Non-normally distributed continuous variables were presented as median and interquartile range [IQR] and compared with Mann-Whitney's test if independent or with Wilcoxon's test in case of dependent variables. Logistic regression model was used to identify predictors and we used a stepwise regression to identify the independent predictors. Odds ratio (OR) with their $95 \%$ confidence interval $(95 \% \mathrm{CI})$ are presented. A $p$ value $<0.05$ was considered as statistically significant.

\section{Results}

\section{Patient characteristics}

The main characteristics of the 405 patients admitted in our Emergency Department due to COVID-19 and then hospitalized in an internal ward are as follows. Median age was 69.8 (IQR 58.6-78.3) years and 68.6\% of patients were males. Underlying cardiovascular disease (hypertension or heart failure or atrial fibrillation or ischemic or valvular heart disease) was highly prevalent (60\%) whereas diabetes was present in $20.2 \%$, pulmonary disease in $14.8 \%$, chronic renal disease in $9.7 \%$ and cancer in $12.1 \%$ of patients.

A total of 172 patients (44.4\%) were treated with ACE inhibitors/ARB/ARNi, and substantial proportion of patients received betablockers, antiarrhythmic drugs (such as amiodarone or flecainide) and antiplatelets or anticoagulants. At hospital arrival, most patients complained of dyspnea and only a small proportion (4\%) reported chest pain.

A positive result on polymerase chain reaction testing of a nasopharyngeal swab was obtained in 321 out of 405 patients $(79.3 \%)$.

Forty $(9.9 \%)$ patients were transferred to ICU with a median time from hospital admission to ICU of 7 days [IQR 4-13.5] and $124(30.6 \%)$ died during hospital stay in a median of 9 days [IQR 5-15] after hospital admission. The total number of patients who died and/or were transferred to ICU during hospital stay was 145 (35.8\%) and this combined outcome occurred in a median of 8 days [IQR 4-11] after hospital admission.

\section{Clinical characteristics according to the hs-Tnl plasma levels (Table 1)}

A hs-TnI value collected within $1 \mathrm{~h}$ after hospital admission was available in 340 patients (84\%). The plasma levels of hs-TnI in the entire cohort was within normal range, with a 95 th percentile of $189.5 \mathrm{pg} / \mathrm{L}$. Elevated hs-TnI values were present in 74 (21.8\%) patients at hospital arrival. The patients with elevated hs-TnI values at hospital arrival were significantly older $(p<0.001)$ and reported more frequently a history of cardiovascular disease $(p=0.006)$ compared to patients with normal hs-TnI values. Concerning laboratory findings and vital signs at hospital admission, patients with elevated hs-TnI values showed significantly higher WBC count $(p=0.01)$, higher creatinine values $(p<0.001)$ and lower $\mathrm{P} / \mathrm{F}$ values $(p=0.017)$ as compared to patients with normal hs-TnI values. Finally, patients with elevated hs-TnI values presented more frequently conduction disturbances $(p=0.002)$, longer QTc interval $(p=0.049)$ or any ECG abnormality $(p=0.048)$ as compared to those with normal hs-TnI values, while atrial fibrillation was similar in the two subgroups.

Death and the composite outcome of death and/or ICU admission occurred in a significantly higher proportion of patients with elevated hs-TnI values at hospital admission as compared to patients with normal hs-TnI values (both $p<0.001$ ). The median time from hospital admission to both the endpoints was similar in the two subgroups.

\section{Clinical characteristics according to positivity to SARS-CoV-2 reverse-transcriptase polymerase chain reaction (RT-PCR) test (Supplementary Table 1)}

Patients with a positive RT-PCR test for SARS-CoV-2 were more frequently males and more frequently had dyspnea at hospital arrival than patients with a negative test. WBC counts were lower, creatinine values were higher and QTc values were longer in patients with positive RT-PCR test. Both death and the combined outcome occurred more frequently in patients with a positive RT-PCR test for SARSCoV-2 ( $p=0.02$ and $p=0.04$, respectively).

\section{Clinical characteristics according to outcome (Table 2)}

Patients who died in hospital were significantly older $(p<0.001)$, had more chronic comorbidities such as cardiovascular disease $(p<0.001)$, chronic renal disease $(p<0.001)$ and diabetes $(p=0.002)$. Patients dying in hospital were more likely to receive drugs such as betablockers $(p<0.001)$ and antiplatelets and/or anticoagulants $(p<0.001)$, whilst there were no significant differences in terms of ACEi/ARB/ARNi assumption $(p=0.15)$. Dyspnea at hospital arrival was more frequently reported by patients who died during the hospitalization $(p=0.009)$, chest pain was equally present $(p=0.98)$. WBC count $(p=0.01)$, platelet count $(p=0.03)$, creatinine values $(p<0.001)$ and hsTnI values $(p<0.001)$ were higher and $\mathrm{P} / \mathrm{F}$ value was lower 
Table 1 Patient characteristics according to hs-TNI plasma levels

\begin{tabular}{|c|c|c|c|}
\hline & hs-TnI $\leq 47(n=266)$ & hs-TnI $>47(n=74)$ & $p$ \\
\hline Age (years), median [IQR] & $68.6[56.4-76.4]$ & $76.1[69.7-82.5]$ & $<0.001$ \\
\hline Males, $n(\%)$ & $188(70.7)$ & $51(68.9)$ & 0.77 \\
\hline \multicolumn{4}{|l|}{ Comorbidities } \\
\hline Cardiovascular disease, $n(\%)$ & $165(63.7)$ & $59(80.8)$ & 0.006 \\
\hline Pulmonary disease, $n(\%)$ & $31(12.1)$ & $13(18.1)$ & 0.19 \\
\hline Chronic renal disease, $n(\%)$ & $19(7.4)$ & $8(11.1)$ & 0.31 \\
\hline Cancer, $n(\%)$ & $31(12.2)$ & $11(15.3)$ & 0.49 \\
\hline Diabetes, $n(\%)$ & $51(20)$ & $17(23.6)$ & 0.51 \\
\hline \multicolumn{4}{|l|}{ Theraphy at admission } \\
\hline $\mathrm{ACEi}$ or $\mathrm{ARB}$ or $\mathrm{ARNi}, n(\%)$ & $114(45.1)$ & $33(46.5)$ & 0.83 \\
\hline Betablockers, $n(\%)$ & $88(34.6)$ & $23(31.9)$ & 0.67 \\
\hline Antiarrhythmics, $n(\%)$ & $9(3.7)$ & $4(5.8)$ & 0.43 \\
\hline Antiplatelets and/or anticoagulants, $n(\%)$ & $86(34)$ & $33(45.8)$ & 0.07 \\
\hline Dyspnea at hospital arrival, $n(\%)$ & $170(65.1)$ & $50(68.5)$ & 0.59 \\
\hline Chest pain at hospital arrival, $n(\%)$ & $8(3.1)$ & $3(4.2)$ & 0.64 \\
\hline \multicolumn{4}{|l|}{ Laboratory findings and vital signs at arrival } \\
\hline $\mathrm{Hb}(\mathrm{g} / \mathrm{dL})$, median [IQR] & $13.5[12.3-14.5]$ & $12.9[11.1-14.8]$ & 0.15 \\
\hline WBC $\left(\times 10^{3} / \mu \mathrm{L}\right)$, median $[\mathrm{IQR}]$ & $6.42[5.02-8.34]$ & $7.45[5.36-11.42]$ & 0.01 \\
\hline $\operatorname{PLT}\left(\times 10^{3} / \mu \mathrm{L}\right)$, median $[\mathrm{IQR}]$ & $180[145-249]$ & $202[138-278]$ & 0.37 \\
\hline Potassium (mEq/L), median [IQR] & $3.96[3.88-4.05]$ & $4.06[3.8-4.48]$ & 0.07 \\
\hline Creatinine (mg/dL), median [IQR] & $0.91[0.73-1.14]$ & $1.09[0.82-1.84]$ & $<0.001$ \\
\hline hsTnI (ng/L), median [IQR] & $11[5-20]$ & $99.5[69-186]$ & $<0.001$ \\
\hline $\mathrm{P} / \mathrm{F}\left(\mathrm{PaO}_{2} / \mathrm{FiO}_{2}\right)$, median [IQR] & 284 [199-336] & $247[120-324]$ & 0.017 \\
\hline Systolic blood pressure (mmHg), median [IQR] & $133[120-145]$ & $138.5[123-150]$ & 0.036 \\
\hline Diastolic blood pressure (mmHg), median [IQR] & 75 [70-85] & $77.5[68-85]$ & 0.79 \\
\hline \multicolumn{4}{|l|}{ ECG findings ${ }^{\mathrm{a}}$} \\
\hline Heart rate (bpm), median [IQR] & 88 [78-99] & $90[76-106]$ & 0.41 \\
\hline Atrial fibrillation, $n(\%)$ & $19(11.5)$ & $6(10.9)$ & 0.9 \\
\hline Conduction disturbances, $n(\%)$ & $42(25.5)$ & $26(47.3)$ & 0.002 \\
\hline PR (ms), median [IQR] & $159[144-180]$ & $170[146-191]$ & 0.14 \\
\hline QTc (ms), median [IQR] & $422[403-448]$ & $441[407-469]$ & 0.049 \\
\hline Repolarization abnormalities, $n(\%)$ & $23(13.9)$ & $14(25.5)$ & 0.048 \\
\hline \multicolumn{4}{|l|}{ Outcomes } \\
\hline Death & $58(21.8)$ & $(38(51.4)$ & $<0.001$ \\
\hline ICU or death & $78(29.3)$ & $38(51.4)$ & $<0.001$ \\
\hline \multicolumn{4}{|l|}{ Time for outcome } \\
\hline Death (days), median [IQR] & $9[6-18]$ & $9[5-15]$ & 0.76 \\
\hline ICU or death (days), median [IQR] & $8[5-11]$ & $7[5-12]$ & 0.9 \\
\hline
\end{tabular}

$A C E i$ ACE inhibitors, $A R B$ angiotensin II receptor blockers, $A R N i$ angiotensin receptor neprilysin inhibitor, $P / F \mathrm{PaO}_{2} / \mathrm{FiO}_{2}, \mathrm{Hb}$ hemoglobin, $W B C$ white blood cell, ICU intensive care unit

${ }^{a}$ ECG data refer to 220 patients, 165 in the hs-TnI $\leq 47$ subgroup and 55 in the hs-TnI $>47$ subgroup $(p<0.001)$ in patients dying in hospital; in addition, it must be emphasized that the median values of hs-TnI were within normal range in non-survivors. Finally, atrial fibrillation was more frequently present in patients who subsequently died in hospital $(p=0.002)$.

When considering in-hospital mortality and/or ICU admission (Table 2-right), patients with the worse prognosis were older $(p<0.001)$ and more frequently had previous history of cardiovascular disease $(p<0.001)$, chronic renal disease $(p<0.001)$ and diabetes $(p=0.008)$. At hospital arrival, dyspnea was highly prevalent $(p=0.01)$, WBC count $(p=0.006)$, platelet count $(p=0.03)$, creatinine values $(p<0.001)$ and hsTnI values $(p<0.001)$ were higher in patients who were later transferred to ICU or died during hospital stay, whilst $\mathrm{P} / \mathrm{F}$ values were lower $(p<0.001)$; importantly, the median values of hs-TnI were within normal 
Table 2 Patient characteristics according to outcome

\begin{tabular}{|c|c|c|c|c|c|c|}
\hline & Survivors $(n=281)$ & Non-survivors $(n=124)$ & $p$ & $\begin{array}{l}\text { Event-free survi- } \\
\text { vors }(n=260)\end{array}$ & $\begin{array}{l}\text { Transferred to ICU } \\
\text { or non-survivors } \\
(n=145)\end{array}$ & $p$ \\
\hline Age (years), median [IQR] & $65[55-75]$ & 76 [70-85] & $<0.001$ & $66[55-76]$ & $74[69-82]$ & $<0.001$ \\
\hline Males, $n(\%)$ & $186(66.2)$ & $92(74.2)$ & 0.11 & $171(65.8)$ & $107(73.8)$ & 0.09 \\
\hline \multicolumn{7}{|l|}{ Comorbidities } \\
\hline Cardiovascular disease, $n(\%)$ & $166(60.4)$ & $102(84.3)$ & $<0.001$ & $151(59.2)$ & $117(83)$ & $<0.001$ \\
\hline Pulmonary disease, $n(\%)$ & $39(14.4)$ & $19(15.7)$ & 0.74 & $38(15)$ & $20(14.5)$ & 0.9 \\
\hline Chronic renal disease, $n(\%)$ & $16(5.9)$ & $22(18.2)$ & $<0.001$ & $15(5.9)$ & $23(16.7)$ & $<0.001$ \\
\hline Cancer, $n(\%)$ & $37(13.8)$ & $10(8.3)$ & 0.12 & $36(14.2)$ & $11(8)$ & 0.07 \\
\hline Diabetes, $n(\%)$ & $43(15.9)$ & $36(29.8)$ & 0.002 & $41(16.2)$ & $38(27.5)$ & 0.008 \\
\hline \multicolumn{7}{|l|}{ Theraphy at admission } \\
\hline $\mathrm{ACEi}$ or $\mathrm{ARB}$ or ARNi, $n(\%)$ & $114(42.1)$ & $58(50)$ & 0.15 & $105(41.3)$ & $67(50.4)$ & 0.09 \\
\hline Betablockers, $n(\%)$ & $79(29)$ & $56(48.3)$ & $<0.001$ & $71(27.8)$ & $64(48.1)$ & $<0.001$ \\
\hline Antiarrhythmics, $n(\%)$ & $8(3.1)$ & $8(7.1)$ & 0.08 & $7(2.9)$ & $9(6.9)$ & 0.07 \\
\hline $\begin{array}{l}\text { Antiplatelets and/or anticoagu- } \\
\text { lants, } n(\%)\end{array}$ & $72(26.6)$ & $71(60.7)$ & $<0.001$ & $67(26.4)$ & $76(56.7)$ & $<0.001$ \\
\hline Dyspnea at hospital arrival, $n(\%)$ & $171(62.2)$ & $93(75.6)$ & 0.009 & $157(61.8)$ & $107(74.3)$ & 0.01 \\
\hline $\begin{array}{l}\text { Chest pain at hospital arrival, } n \\
(\%)\end{array}$ & $11(68.7)$ & $5(4.1)$ & 0.98 & $10(4)$ & $6(4.2)$ & 0.92 \\
\hline \multicolumn{7}{|c|}{ Laboratory findings and vital signs at arrival } \\
\hline $\mathrm{Hb}(\mathrm{g} / \mathrm{dL})$, median $[\mathrm{IQR}]$ & $13.5[12.3-14.4]$ & $12.9[11.8-14.5]$ & 0.13 & $13.5[12.3-14.5]$ & 13 [12-14.3] & 0.19 \\
\hline $\mathrm{WBC}\left(\times 10^{3} / \mu \mathrm{L}\right)$, median $[\mathrm{IQR}]$ & $6.3[5-8]$ & $7.5[5-10.3]$ & 0.01 & $6.2[5-8]$ & $7.5[5.2-10.2]$ & 0.006 \\
\hline $\operatorname{PLT}\left(\times 10^{3} / \mu \mathrm{L}\right)$, median $[\mathrm{IQR}]$ & $187.5[149-257]$ & 174 [129-227] & 0.03 & $188[150-258]$ & $174[130-230]$ & 0.03 \\
\hline $\begin{array}{l}\text { Potassium }(\mathrm{mEq} / \mathrm{L}), \text { median } \\
\text { [IQR] }\end{array}$ & $3.9[3.6-4.3]$ & $4.1[3.8-4.5]$ & 0.006 & $3.9[3.6-4.4]$ & $4.1[3.8-4.4]$ & 0.02 \\
\hline $\begin{array}{l}\text { Creatinine }(\mathrm{mg} / \mathrm{dL}) \text {, median } \\
\text { [IQR] }\end{array}$ & $0.88[0.72-1.1]$ & $1.09[0.88-1.61]$ & $<0.001$ & $0.87[0.71-1.1]$ & $1.05[0.86-1.55]$ & $<0.001$ \\
\hline hsTnI (ng/L), median [IQR] & $11[5-26]$ & $31[15-80]$ & $<0.001$ & $11[5-27]$ & $27[12-62]$ & $<0.001$ \\
\hline $\mathrm{P} / \mathrm{F}\left(\mathrm{PaO}_{2} / \mathrm{FiO}_{2}\right)$, median [IQR] & $296[220-351]$ & $231[117-316]$ & $<0.001$ & 297 [233-347] & $230[120-330]$ & $<0.001$ \\
\hline $\begin{array}{l}\text { Systolic blood pressure }(\mathrm{mmHg}) \\
\text { median [IQR] }\end{array}$ & $135[120-145]$ & $130[120-149]$ & 0.68 & 134 [120-145] & $130[120-147]$ & 0.73 \\
\hline $\begin{array}{l}\text { Diastolic blood pressure } \\
(\mathrm{mmHg}), \text { median }[\mathrm{IQR}]\end{array}$ & $80[70-85]$ & $73[65-80]$ & 0.003 & $80[70-86]$ & $74[65-80]$ & 0.002 \\
\hline \multicolumn{7}{|l|}{ ECG findings } \\
\hline Heart rate (bpm), median [IQR] & 88 [77-99] & $88[78-100]$ & 0.7 & 88 [77-99] & $87[77-100]$ & 0.94 \\
\hline Atrial fibrillation, $n(\%)$ & $12(6.9)$ & $17(20.2)$ & 0.002 & $12(7.1)$ & $17(19.3)$ & 0.003 \\
\hline Conduction disturbances, $n(\%)$ & $51(29)$ & $31(36)$ & 0.25 & $49(28.5)$ & $33(36.7)$ & 0.18 \\
\hline PR (ms), median [IQR] & 154 [142-172] & 175 [157-190] & $<0.001$ & $154[142-172]$ & $173[157-190]$ & $<0.001$ \\
\hline QTc (ms), median [IQR] & $425[406-450]$ & $436[410-460]$ & 0.12 & $426[406-450]$ & $434[411-460]$ & 0.12 \\
\hline $\begin{array}{l}\text { Repolarization abnormalities, } \\
n(\%)\end{array}$ & $28(15.9)$ & $20(23.3)$ & 0.15 & $28(16.3)$ & $20(22.2)$ & 0.24 \\
\hline
\end{tabular}

Abbreviations as in Table 1

range in patients who died during hospital stay or were transferred to ICU. Atrial fibrillation as presenting rhythm was more frequent in those patients dying or ending up at the $\operatorname{ICU}(p=0.003)$.

The analysis of patients for whom both hs-TnI and ECG were available showed that the outcome progressively worsened when the number of parameters that we included in the definition of cardiac involvement increased (Fig. 1). This correlation was valid considering both in-hospital death (from $7.9 \%$ in patients without cardiac involvement to $61.5 \%$ in patients with all three parameters indicative of cardiac involvement) and the composite outcome of death and/or ICU admission (from $9.5 \%$ in patients without cardiac involvement to $61.5 \%$ in patients with high-grade cardiac involvement). 


\section{Predictors of death and of the composite outcome}

Logistic regression analysis showed that elevated hs-TnI values ( $p=<0.001$ ), presence of ECG abnormalities and/or $\mathrm{AF}$ at ECG $(p=0.01)$ and history of cardiovascular disease $(p<0.001)$ were significant predictors of in-hospital death at univariate analysis, alongside older age $(p<0.001)$ and $\mathrm{P} / \mathrm{F}$ less than $200(p<0.001)$. However, only age $(p<0.001)$, elevated hs-TnI values $(p=0.009)$ and $\mathrm{P} / \mathrm{F}(p=0.014)$ were independent predictors at multivariable analysis (Table $3-$ upper part). There was no interaction between the result of RT-PCR test for SARS-CoV-2 and death $(p=0.87)$.

Considering the combined outcome of death and/or transfer to ICU, elevated hs-TnI values $(<0.001)$, presence of ECG abnormalities and/or AF at ECG $(p=0.025)$, history of cardiovascular disease $(p<0.001)$, older age $(p<0.001)$ and $\mathrm{P} / \mathrm{F}$ less than 200 at hospital arrival $(p<0.001)$ were predictor at univariable analysis but, again, only age $(p<0.001), \mathrm{P} / \mathrm{F}$ less than $200(p=0.001)$ and elevated hs-TnI values $(p=0.032)$, were independent predictors at multivariable analysis (Table 3-lower part). There was no interaction between the result of RT-PCR test for SARS-CoV-2 and the combined outcome $(p=0.94)$.

\section{Discussion}

This study reports the first large case series of consecutively hospitalized patients with COVID-19 in an academic hospital in Lombardy, which is one of the most affected Italian and worldwide regions by the COVID19 epidemic, both in terms of number of patients needing admission and in terms of fatality rate. The main finding is that, in this cohort, in-hospital mortality
Fig. 1 Death rate and ICU or death rate according to the presence of hsTNI plasma levels and/or any electrocardiographic abnormality and/or history of cardiovascular disease

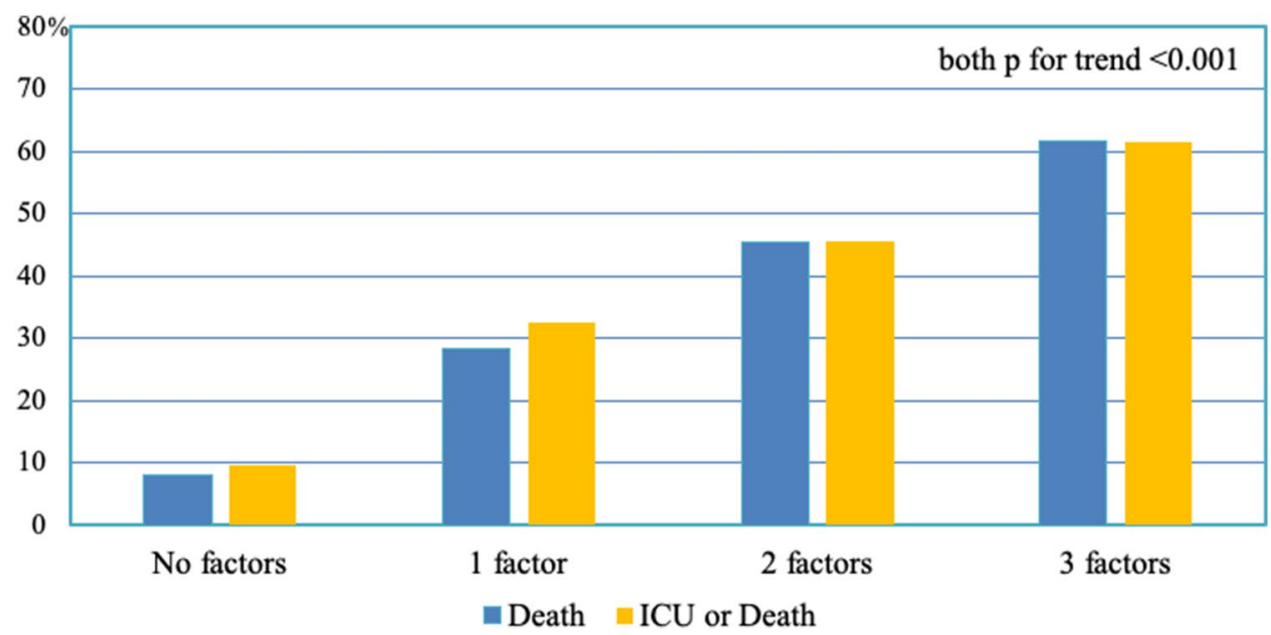

Table 3 Univariable and multivariable predictors of death or of the combination of death and/or ICU admittance

\begin{tabular}{|c|c|c|c|c|}
\hline & Univariable analysis & $p$ & Multivariable analysis & $p$ \\
\hline \multicolumn{5}{|l|}{ Death } \\
\hline Age & 1.09 (95\% CI 1.07-1.12) & $<0.001$ & 1.09 (95\% CI 1.06-1.14) & $<0.001$ \\
\hline $\mathrm{P} / \mathrm{F}<200$ & 2.89 (95\% CI 1.76-4.71) & $<0.001$ & $2.53(95 \%$ CI $1.21-5.31)$ & 0.014 \\
\hline Elevated hs-TnI values & 3.78 (95\% CI 2.2-6.5) & $<0.001$ & $2.74(95 \%$ CI $1.28-5.84)$ & 0.009 \\
\hline ECG abnormalities and/or AF at ECG & $2.11(95 \%$ CI $1.19-3.74)$ & 0.010 & - & \\
\hline History of cardiovascular disease & $3.52(95 \%$ CI $2.04-6.08)$ & $<0.001$ & - & \\
\hline \multicolumn{5}{|l|}{ ICU or death } \\
\hline Age & $1.06(95 \%$ CI $1.04-1.08)$ & $<0.001$ & 1.09 (95\% CI 1.05-1.13) & $<0.001$ \\
\hline $\mathrm{P} / \mathrm{F}<200$ & 3.49 (95\% CI 2.15-5.65) & $<0.001$ & $3.11(95 \%$ CI 1.51-6.4) & 0.002 \\
\hline Elevated hs-TnI values & $2.54(95 \%$ CI $1.5-4.31)$ & $<0.001$ & 2.27 (95\% CI 1.07-4.8) & 0.032 \\
\hline ECG abnormalities and/or AF at ECG & $1.92(95 \%$ CI 1.09-3.38) & 0.025 & - & \\
\hline History of cardiovascular disease & $3.36(95 \%$ CI $2.03-5.57)$ & $<0.001$ & - & \\
\hline
\end{tabular}

Abbreviations as in Table 1 
was associated with older age, respiratory failure and elevated plasma levels of hs-TnI whereas cardiovascular comorbidities were not an independent risk factor at multivariable analysis. Importantly, plasma levels of hs-TnI were, on average, within normal range in non-survivors.

\section{Cardiac involvement and outcome in COVID-19}

Since the onset of the pandemic, a growing body of evidence has shown that a substantial proportion of COVID-19 patients may have cardiovascular complications, up to cardiac arrest [18]. However, it is still a matter of debate whether the presence of elevated hsTnI plasma levels and/or of cardiovascular comorbid conditions at hospital presentation poses an independent risk in such patients or whether the risk is mediated by other factors. In the present series, cardiac involvement upon admission was associated with a higher risk of events during the subsequent hospital stay at univariable analysis. The mortality rate and the rate of the combined endpoint were more than fivefold greater in patients with elevated hs-TnI plasma levels, electrocardiographic abnormality and history of cardiovascular disease as compared to those without any of these parameters. However, when age and respiratory failure at hospital admission were analyzed together with cardiac involvement in a multivariable analysis, only older age, a P/F ratio $<200$ and elevated hs-TnI plasma levels were significant predictor of death and of the combined outcome. Importantly, the hs-TnI plasma levels were generally low and in the normal range even in non-surviving patients. This datum, together with the observation that patients with elevated hs-TnI values at admission had laboratory and respiratory function data indicative of a more severe systemic infection, would support hypothesis that the increase of hs-TnI levels in COVID-19 patients is mainly the consequence of a more severe systemic disease rather being indicative of primary cardiac injury, as already described in the literature [19].

Among the parameters which did not result in statistically significant predictors of events are home therapy with angiotensin converting enzyme inhibitors (ACEi) and angiotensin receptor blockers (ARB). Concerns about ACEI/ARB have been raised since angiotensin converting enzyme 2 (ACE2) is a potential target for COVID-19 infection. However, although evidence regarding the use of ACEI/ ARB in patients with COVID-19 infection is still emerging, our findings seem to be consistent with the latest studies published on this topic $[20,21]$.

\section{Mortality rate}

It is important to observe that the fatality rate according to cardiovascular involvement in the present cohort is substantially similar to what has been reported in previous studies, ranging from below $10 \%$ for subgroups without cardiac injury to greater than $50 \%$ for patients with cardiac injury or history of cardiovascular disease. Nonetheless, in the present population, the overall hospital mortality rate was higher compared to previous series [4-7, 22, 23]. The reasons for this may be different. First, the median age in the present cohort of patients is 70 years, greater than in previously reported series, and older age has been shown to be crucial in all studies published so far. Another contributing factor could be the high prevalence of comorbidities in particular when compared to Asian populations. Elderly patients with multimorbidity are usually frail and more likely to have worse outcomes, as already shown in different hospital settings [24]. Moreover, the present cohort was characterized by a substantial pulmonary involvement, in particular considering that the most critical patients, i.e. those who were directly admitted to the ICU, were not included in the present series; this is likely to be a crucial determinant for the dismal prognosis of the present cohort in Pavia. The clinical implication is that COVID-19 patients should possibly be treated earlier, rather than delaying hospital arrival until severe respiratory symptoms have developed. Finally, to the best of our knowledge, the present population is the first one composed almost exclusively of Caucasian patients; it cannot be excluded that ethnicity may play a role in fatality rate and further studies should be focused to address this issue.

\section{Limitations}

The main limitation of the present study is its retrospective design; in particular, it lacks echocardiographic imaging of the patients and an in-depth analysis of markers of inflammation such as interleukin-6 which are not routinely performed in the Emergency Department. In addition, how treatment during hospitalization has influenced the outcome has not been analyzed but it was out of the scope of the study. The number of patients enrolled, although quite large for a single center and similar to those included in previous publications, is still too low to allow a differentiation among several types of heart diseases or of ECG abnormalities. This might probably be performed in future metanalysis. We found differences between the baseline characteristics of patients with a positive RT-PCR test for SARS-CoV-2 and those of patients with a negative test. There can be different reasons for these findings, which are out of the scope of our study, but which deserve to be further investigated in future studies.

Finally, considering the retrospective design of the study, unmeasured confounding may be present. 


\section{Conclusions}

Myocardial injury at presentation was associated with poor prognosis in COVID-19 patients, but, even in a population of patients who did not require invasive ventilation at hospital admission, mortality was mainly driven by older age and respiratory failure.

Acknowledgements Stefano Ghio, Luigi Oltrona Visconti, Enrico Baldi, Marco Ferlini, Massimiliano Gnecchi, Alessandra Greco, Giulia Magrini, Laura Scelsi, Rossana Totaro, Alessandro Vicentini, Mauro Acquaro, Michela Coccia, Sabato D'Amore, Simonluca Digiacomo Davide Foglia, Francesco Jeva, Lucrezia Masiello, Claudio Montalto, Martina Moschella, Laura Pezza, Stefano Perlini, Claudia Alfano, Marco Bonzano, Federica Briganti, Giuseppe Crescenzi, Anna Giulia Falchi, Elena Maggi, Roberta Guarnone, Barbara Guglielmana, Ilaria Francesca Martino, Maria Serena Pioli Di Marco, Pietro Pettenazza, Federica Quaglia, Anna Sabena, Francesco Salinaro, Francesco Speciale, Ilaria Zunino, Giulia Sturniolo, Federico Bracchi, Elena Lago, Angelo Corsico, Davide Piloni, Giulia Accordino, Cecilia Burattini, Antonio Di Sabatino, Marco Vincenzo Lenti, Ivan Pellegrino, Simone Soriano, Giovanni Santacroce, Alessandro Parodi, Federica Borrelli de Andreis, Raffaele Bruno, Angela Di Matteo, Elena Maria Seminari, Valentina Zuccaro, Francesco Moioli, Guido Tavazzi, Valentino Dammassi, Riccardo Albertini, and Catherine Klersy.

Investigators and co-authors of the San Matteo COVID Cardiac Injury Task Force are as follows: Division of Cardiology: Stefano Ghio, Luigi Oltrona Visconti, Enrico Baldi, Marco Ferlini, Massimiliano Gnecchi, Alessandra Greco, Giulia Magrini, Laura Scelsi, Rossana Totaro, Alessandro Vicentini, Mauro Acquaro, Michela Coccia, Sabato D'Amore, Simonluca Digiacomo Davide Foglia, Francesco Jeva, Lucrezia Masiello, Claudio Montalto, Martina Moschella, Laura Pezza. Emergency Department: Stefano Perlini, Claudia Alfano, Marco Bonzano, Federica Briganti, Giuseppe Crescenzi, Anna Giulia Falchi, Elena Maggi, Roberta Guarnone, Barbara Guglielmana, Ilaria Francesca Martino, Maria Serena Pioli Di Marco, Pietro Pettenazza, Federica Quaglia, Anna Sabena, Francesco Salinaro, Francesco Speciale, Ilaria Zunino, Giulia Sturniolo, Federico Bracchi, Elena Lago. Division of Respiratory Diseases: Angelo Corsico, Davide Piloni, Giulia Accordino, Cecilia Burattini. Division of Internal Medicine: Antonio Di Sabatino, Marco Vincenzo Lenti, Ivan Pellegrino, Simone Soriano, Giovanni Santacroce, Alessandro Parodi, Federica Borrelli de Andreis. Division of Infectious Disease: Raffaele Bruno, Angela Di Matteo, Elena Maria Seminari, Valentina Zuccaro. Intensive Care Unit: Francesco Moioli, Guido Tavazzi, Valentino Dammassi. Clinical Chemistry Laboratory: Riccardo Albertini. Service of Clinical Epidemiology and Biometry: Catherine Klersy.

Author contributions SG, EB, LOV and SP planned the study; SG and EB drafted the manuscript; ML, AD, ADM, VZ, DP, AC, MG, FS, SG, $\mathrm{EB}, \mathrm{LOV}$, and SP gave substantial contributions to acquisition of data, analysis and interpretation of data, revised the manuscript critically for important intellectual content and provided final approval of the version to be published. All the authors read and approved the final version. SG is responsible for the overall content as guarantor. All above authors take responsibility for all aspects of the reliability and freedom from bias of the data presented and their discussed interpretation.

Funding The study was not funded.

\section{Compliance with ethical standards}

Conflict of interest The authors declare that they have no conflict of interest.

Human and animal rights All procedures performed in studies involving human participants were in according with the ethical standard.

Informed consent The review board of the Fondazione Policlinico San Matteo approved the publication of anonymized case series of COVID19 patients using data collected for routine clinical practice and waived the requirement for a specific informed consent.

\section{References}

1. Zhu N, Zhang D, Wang W, China Novel Coronavirus Investigating, and Research Team et al (2020) A novel coronavirus from patients with pneumonia in China, 2019. N Engl J Med 382:727-733

2. Bangalore S, Sharma A, Slotwiner A et al (2020) ST-segment elevation in patients with COVID-19-a case series. N Engl J Med 382:2478-2480

3. Gnecchi M, Moretti F, Bassi EM et al (2020) Myocarditis in a 16-year-old boy positive for SARS-CoV-2. Lancet 395(10242): 116

4. Shi S, Qin M, Shen B et al (2020) Association of cardiac injury with mortality in hospitalized patients with COVID-19 in Wuhan, China. JAMA Cardiol 5:802-810

5. Guo T, Fan Y, Chen M et al (2020) Cardiovascular implications of fatal outcomes of patients with coronavirus disease 2019 (COVID-19). JAMA Cardiol 5:1-8

6. Wei JF, Huang FY, Xiong TY et al (2020) Acute myocardial injury is common in patients with COVID-19 and impairs their prognosis. Heart 106:1154-1159. https://doi.org/10.1136/heartjnl-2020317007

7. Inciardi RM, Adamo M, Lupi L, Cani DS, Di Pasquale M, Tomasoni D, Italia L, Zaccone G, Tedino C, Fabbricatore D, Curnis A, Faggiano P, Gorga E, Lombardi CM, Milesi G, Vizzardi E, Volpini M, Nodari S, Specchia C, Maroldi R, Bezzi M, Metra M (2020) Characteristics and outcomes of patients hospitalized for COVID-19 and cardiac disease in Northern Italy. Eur Heart J 41:1821-1829

8. Zhou F, Yu T, Du R et al (2020) Clinical course and risk factors for mortality of adult inpatients with COVID-19 in Wuhan, China: a retrospective cohort study. Lancet 395:1054-1062

9. Lippi G, Lavie CJ, Sanchis-Gomar F (2020) Cardiac troponin I in patients with coronavirus disease 2019 (COVID-19): evidence from a meta-analysis. Prog Cardiovasc Dis. https://doi. org/10.1016/j.pcad.2020.03.001

10. Huang C, Wang Y, Li X et al (2020) Clinical features of patients infected with 2019 novel coronavirus in Wuhan, China. Lancet 395:497-506

11. Liu Y, Yang Y, Zhang C et al (2020) Clinical and biochemical indexes from 2019-nCoV infected patients linked to viral loads and lung injury. Sci China Life Sci 63:364-374

12. Wang $\mathrm{D}, \mathrm{Hu} \mathrm{B}, \mathrm{Hu} \mathrm{C}$ et al (2020) Clinical characteristics of 138 hospitalized patients with 2019 novel coronavirus-infected pneumonia in Wuhan, China. JAMA 323:1061-1069

13. Ruan Q, Yang K, Wang W et al (2020) Clinical predictors of mortality due to COVID-19 based on an analysis of data of 150 patients from Wuhan, China. Intensive Care Med 46:846-848 
14. Cheng R, Leedy D (2020) COVID-19 and acute myocardial injury: the heart of the matter or an innocent bystander? Heart 106:1122-1124. https://doi.org/10.1136/heartjnl-2020-317025

15. Song CY, Yang DG, Lu YQ (2020) A COVID-19 patient with seven consecutive false-negative rRT-PCR results from sputum specimens. Intern Emerg Med 2:1-4. https://doi.org/10.1007/ s11739-020-02423-y

16. Sciacqua A, Pujia R, Arturi F, Hribal ML, Montalcini T (2020) COVID-19 and elderly: beyond the respiratory drama. Intern Emerg Med. https://doi.org/10.1007/s11739-020-02424-x

17. Kavsak PA, Worster A, Hill SA et al (2018) Evaluation of the Siemens ADVIA Centaur high-sensitivity cardiac troponin I assay in serum. Clin Chim Acta 487:216-221

18. Baldi E, Sechi GM, Mare C et al (2020) COVID-19 kills at home: the close relationship between the epidemic and the increase of out-of-hospital cardiac arrests. Eur Heart J. https:// doi.org/10.1093/eurheartj/ehaa508

19. Korff S, Katus HA, Giannitsis E (2006) Differential diagnosis of elevated troponins. Heart 92:987-993

20. Mancia G, Rea F, Ludergnani M et al (2020) Renin-angiotensinaldosterone system blockers and the risk of Covid-19. N Engl J Med. https://doi.org/10.1056/NEJMoa2006923
21. Albini A, Di Guardo G, Noonan DM, Lombardo M (2020) The SARS-CoV-2 receptor, ACE-2, is expressed on many different cell types: implications for ACE-inhibitor- and angiotensin II receptor blocker-based cardiovascular therapies. Intern Emerg Med. https ://doi.org/10.1007/s11739-020-02364-6

22. Richardson S, Hirsch JS, Narasimhan M, Northwell COVID19 Research Consortium et al (2020) Presenting characteristics, comorbidities, and outcomes among 5700 patients hospitalized with COVID-19 in the New York City Area. JAMA 323:2052-2059

23. Wu JT, Leung K, Bushman M et al (2020) Estimating clinical severity of COVID-19 from the transmission dynamics in Wuhan, China. Nat Med 26:506-510

24. Corazza GR, Formagnana P, Lenti MV (2019) Bringing complexity into clinical practice: an internistic approach. Eur J Intern Med 61:9-14

Publisher's Note Springer Nature remains neutral with regard to jurisdictional claims in published maps and institutional affiliations.

\section{Affiliations}

\section{Stefano Ghio ${ }^{1}$ - Enrico Baldi ${ }^{2,3}$ - Alessandro Vicentini ${ }^{2} \cdot$ Marco Vincenzo Lenti $^{4}$. Antonio Di Sabatino ${ }^{4}$. Angela Di Matteo ${ }^{5}$. Valentina Zuccaro ${ }^{5}$. Davide Piloni ${ }^{6}$. Angelo Corsico ${ }^{6}$. Massimiliano Gnecchi $^{2,3}$. Francesco Speciale ${ }^{7,8}$. Anna Sabena ${ }^{7,8} \cdot$ Luigi Oltrona Visconti $^{1}$. Stefano Perlini ${ }^{7,8}$ on behalf of San Matteo COVID Cardiac Injury Task Force}

1 Division of Cardiology, Fondazione IRCCS Policlinico San Matteo, Viale Golgi 19, 27100 Pavia, Italy

2 Cardiac Intensive Care Unit, Arrhythmia and Electrophysiology, Laboratory of Clinical and Experimental Cardiology, Fondazione IRCCS Policlinico San Matteo, Pavia, Italy

3 Cardiology Unit, Department of Molecular Medicine, University of Pavia, Pavia, Italy

4 Department of Internal Medicine, Fondazione IRCCS Policlinico San Matteo, Pavia, University of Pavia, Pavia, Italy
5 Division of Infectious Disease, Fondazione IRCCS Policlinico San Matteo, Pavia, University of Pavia, Pavia, Italy

6 Division of Respiratory Diseases, Fondazione IRCCS Policlinico San Matteo, Pavia, University of Pavia, Pavia, Italy

7 Emergency Department, Fondazione IRCCS Policlinico San Matteo, Pavia, Pavia, Italy

8 Department of Internal Medicine, University of Pavia, Pavia, Italy 